\title{
STRAIGHT LINE EMBEDDINGS OF CUBIC PLANAR GRAPHS WITH INTEGER EDGE LENGTHS
}

\author{
JIM GEELEN, ANJIE GUO, AND DAVID MCKINNON
}

\begin{abstract}
We prove that every simple cubic planar graph admits a planar embedding such that each edge is embedded as a straight line segment of integer length.
\end{abstract}

\section{INTRODUCTION}

We consider only simple finite graphs. A straight line embedding of a graph $G=(V, E)$ is an injective function $\phi: V \rightarrow \mathbb{R}^{2}$ such that for any two distinct edges $a b, c d \in E$ the straight line segments $(\phi(a), \phi(b))$ and $(\phi(c), \phi(d))$ are internally disjoint (that is, they may only meet at their ends). It is a well know classical result that every planar graph admits a straight line embedding; see, for example, Wagner [7] or Fáry [3]. Given a straight line embedding of $G$, the length of an edge $u v \in E$ is the Euclidean distance between $\phi(u)$ and $\phi(v)$, which we denote by $\operatorname{dist}(\phi(u), \phi(v))$.

In this paper we address a special case of the following conjecture of Kennitz and Harborth [5, 4]; see also the book by Brass, Moser, and Pach [1].

Conjecture 1.1. Every planar graph admits a straight line embedding with integer edge lengths.

Note that, up to scaling, it suffices to find a straight line embedding with rational edge lengths. We prove Conjecture 1.1 for the class of cubic planar graphs. (A graph is cubic if each of its vertices has degree 3.) The result for cubic planar graphs relies on the following result for general cubic graphs.

Theorem 1.2. Let $G=(V, E)$ be a cubic graph, let $\phi: V \rightarrow \mathbb{R}^{2}$, and let $\epsilon>0$. Then there exists a function $\psi: V \rightarrow \mathbb{Q}^{2}$ such that

Date: January 10, 2008.

1991 Mathematics Subject Classification. 05B35.

Key words and phrases. planar graphs, straight-line embedding.

This research was partially supported by grants from the Natural Sciences and Engineering Research Council of Canada. 
1. $\operatorname{dist}(\psi(u), \psi(v)) \in \mathbb{Q}$ for each $u v \in E$, and

2. $\operatorname{dist}(\psi(v), \phi(v)) \leq \epsilon$ for each $v \in V$.

\section{Preliminaries}

We require the following two theorems that are both of interest in their own right.

Theorem 2.1 (Berry [2]). If $A, B, C \in \mathbb{R}^{2}$ are non-collinear points such that $\operatorname{dist}(A, B)$, $\operatorname{dist}(A, C)^{2}$, and $\operatorname{dist}(B, C)^{2}$ are rational, then the set of points that are a rational distance from each of $A, B$, and $C$ forms a dense subset of $\mathbb{R}^{2}$.

Berry also notes that there are no points in the plane at rational distance from the three vertices of a triangle with sides $\sqrt{2}, \sqrt{3}$, and $\sqrt{5}$, so the condition that one side is rational is unavoidable.

Theorem 2.2. If $A, B, C \in \mathbb{Q}^{2}$ are not collinear and $x \in \mathbb{R}^{2}$ such that $\operatorname{dist}(x, y)^{2}$ is rational for each $y \in\{A, B, C\}$, then $x \in \mathbb{Q}^{2}$.

Proof. By possibly translating the points, we may assume that $C=$ $(0,0)$. Let $\alpha=\left(A_{1}-x_{1}\right)^{2}+\left(A_{2}-x_{2}\right)^{2}, \beta=\left(B_{1}-x_{1}\right)^{2}+\left(B_{2}-x_{2}\right)^{2}$, and $\gamma=x_{1}^{2}+x_{2}^{2}$. Thus $\alpha=\operatorname{dist}(x, A)^{2}, \beta=\operatorname{dist}(x, B)^{2}$, and $\gamma=\operatorname{dist}(x, C)^{2}$ are all rational. Note that

$$
\begin{aligned}
A_{1} x_{1}+A_{2} x_{2} & =\frac{1}{2}\left(\gamma+A_{1}^{2}+A_{2}^{2}-\alpha\right) \\
B_{1} x_{1}+B_{2} x_{2} & =\frac{1}{2}\left(\gamma+B_{1}^{2}+B_{2}^{2}-\beta\right) .
\end{aligned}
$$

Since $A, B$, and $C$ are not collinear, $A$ is not a scalar multiple of $B$. Considering $x_{1}$ and $x_{2}$ as variables, we have two rational linear equations with a unique rational solution. Therefore $x$ is rational.

\section{The Main Results}

We are interested in graphs $G=(V, E)$ satisfying:

Property 3.1. For any function $\phi: V \rightarrow \mathbb{R}^{2}$ and any real number $\epsilon>0$, there exists a function $\psi: V \rightarrow \mathbb{Q}^{2}$ such that

1. $\operatorname{dist}(\psi(u), \psi(v)) \in \mathbb{Q}$ for each $u v \in E$, and

2. $\operatorname{dist}(\psi(v), \phi(v)) \leq \epsilon$ for each $v \in V$.

Lemma 3.2. Let $z$ be a vertex of degree 3 in a simple graph $G=(V, E)$ and let $a, b$, and $c$ be the three neighbours of $z$. If $a b \in E$ and $G-z$ satisfies Property 3.1, then $G$ satisfies Property 3.1. 
Proof. Let $\phi: V \rightarrow \mathbb{R}^{2}$ and $\epsilon>0$. By possibly perturbing $\phi$ and adjusting $\epsilon$ accordingly, we may assume that $\phi$ is injective and that the image of $\phi$ does not contain three collinear points. Moreover, by possibly further decreasing $\epsilon$, we may assume that there do not exist three collinear points $x_{1}, x_{2}, x_{3} \in \mathbb{R}^{2}$ with $\operatorname{dist}\left(x_{i}, \phi\left(u_{i}\right)\right) \leq \epsilon$ for $i \in$ $\{1,2,3\}$.

Since $G-z$ satisfies Property 3.1, there is a function $\psi: V-\{z\} \rightarrow \mathbb{Q}^{2}$ such that

1. $\operatorname{dist}(\psi(u), \psi(v)) \in \mathbb{Q}$ for each $u v \in E(G-z)$, and

2. $\operatorname{dist}(\psi(v), \phi(v)) \leq \epsilon$ for each $v \in V-\{z\}$.

Note that $\psi(a), \psi(b), \psi(c) \in \mathbb{Q}^{2}$ and, since $a b \in E$, $\operatorname{dist}(\psi(a), \psi(b))$ is rational. Hence $\operatorname{dist}(\psi(a), \psi(b))$, $\operatorname{dist}(\psi(b), \psi(c))^{2}$, and $\operatorname{dist}(\psi(a), \psi(c))^{2}$ are all rational. Therefore, by Theorem 2.1, there is a point $x \in \mathbb{R}^{2}$ with $\operatorname{dist}(x, \psi(z)) \leq \epsilon$ that is at a rational distance from each of $\phi(a), \phi(b)$, and $\phi(c)$. By Lemma 2.2, the point $x$ is rational. Now extend $\phi$ to a function $\phi: V \rightarrow \mathbb{Q}^{2}$ by defining $\phi(z)=x$. This shows that $G$ satisfies Property 3.1, as required.

We are ready to prove Theorem 1.2. Our original proof was somewhat more convoluted, the simpler version presented here was suggested by a referee. We restate a mild strengthening of the result to facilitate induction.

Theorem 3.3. Every simple graph with maximum degree $\leq 3$ satisfies Property 3.1.

Proof. Suppose that the result is false and let $G=(V, E)$ be a counterexample with $|V|$ minimum. Let $z \in V$. The case that $z$ has degree $\leq 2$ is straightforward, so we may assume that $z$ has degree 3 . Let $a$, $b$ and $c$ be the three neighbours of $z$. Let $G^{\prime}=\left(V, E^{\prime}\right)$ be the simple graph obtained from $G$ by adding the edge $a b$ (if $a b$ was already an edge of $G$, then $G^{\prime}=G$ ). Observe that $G^{\prime}-z$ has maximum degree $\leq 3$. Then, since $G$ is a minimum counterexample, $G^{\prime}-z$ satisfies Property 3.1. By Lemma 3.2, $G^{\prime}$ satisies Property 3.1. Since $G$ is a subgraph of $G^{\prime}, G$ also satisfies Property 3.1. This contradicts that fact that $G$ is a counterexample and, hence, the result holds.

Corollary 3.4. Every cubic planar graph admits a straight line embedding with integer edge lengths.

Proof. Let $\phi: V \rightarrow \mathbb{R}^{2}$ be a straight line embedding of a cubic planar graph $G=(V, E)$. Note that $\phi$ remains a straight line embedding under arbitrarily small perturbations. That is, there exists $\epsilon>0$ such that, if $\psi: V \rightarrow \mathbb{R}^{2}$ is a function satisfying $\operatorname{dist}(\phi(v), \psi(v))$ for each 
$v \in V$, then $\psi$ is a straight line embedding of $G$. By Theorem 1.2 there is a straight line embedding $\psi$ of $G$ with rational edge lengths. A suitable scaling of $\psi$ gives integer edge lengths.

\section{Concluding Remarks}

We do not know of a graph that does not satisfy Property 3.1, but it seems likely that such graphs exist. It seems reasonable to conjecture that all planar graphs satisfy the property. Using Lemma 3.2 it is easy to show that graphs of tree-width 3 satisfy the property. Thus planar graphs of tree-width 3 satisfy Conjecture 1.1; this result is already implicit in Kemnitz and Harborth [5].

Property 3.1 is also of interest for small complete graphs, particularly $K_{8}$. A famous problem of Erdös asks: How many points we can find in the plane with pairwise rational distances such that no three are on a line and no four are on a circle? A collection of 7 such points has recently been discovered by Kreisel and Kurz [6], but the problem remains open for 8 .

Theorem 2.1 plays a crucial role in our proof of Theorem 1.2. This suggests the following question.

Problem 4.1. Let $A, B_{1}, \ldots, B_{k} \in \mathbb{Q}^{2}$ such that no three of these points are collinear and $\operatorname{dist}\left(A, B_{i}\right)$ is rational for each $i \in\{1, \ldots, k\}$. Does the set of points that are a rational distance from each of $A, B_{1}, \ldots, B_{k}$ form a dense subset of $\mathbb{R}^{2}$ ?

An affirmative answer to Problem 4.1 for $k=4$ would prove that planar graphs satisfy Property 3.1 and, hence, would verify Conjecture 1.1 .

\section{ACKNOWLEDGMENT}

The proof of Theorem 1.2 is much clearer due to a suggestion of a referee.

\section{REFERENCES}

[1] P. Brass, W.O.J. Moser, and J. Pach, "Research problems in Discrete Geometry", Springer-Verlag, 2005.

[2] T.G. Berry. "Points at rational distances from the vertices of a triangle," Acta Arith. 62 (1992), 391-398.

[3] I. Fáry, "On straight line representations of planar graphs," Acta Sci. Math. (Szeged) 11 (1948), 229-233. 
[4] H. Harborth, A. Kemnitz, M. Möller, and A. Süssenbach, "Ganzzahlige planare Darstellungen der platonischen Körper," Elem. Math. 42 (1987), 118-122.

[5] A. Kemnitz and H. Harborth, "Plane integral drawings of planar graphs", Discrete Math. 236 (2001), 191-195.

[6] T. Kreisel and S. Kurz, "There are integral heptagons, no three points on a line, no four on a circle," preprint, November 2006.

[7] K. Wagner, "Bemerkungen zum Vierfarbensatz," Jarhesber. Deutsch. Math. Verin 46 (1936), 26-32.

Department of Combinatorics and Optimization, University of Waterloo, Waterloo, Canada N2L 3G1

Undergraduate student, Faculty of Mathematics, University of Waterloo, Waterloo, Canada N2L 3G1

Department of Pure Mathematics, University of Waterloo, WaterLoo, Canada N2L 3G1 